ORIGINAL ARTICLE

\title{
Control of trunk muscles activity while manual material handling symmetrically and asymmetrically, Based on Motor control strategy
}

\author{
MASOUD GHOFRANI ${ }^{1}$, MANIJEH SOLEIMANIFAR ${ }^{1,2}{ }^{*}$, SAEED TALEBIAN ${ }^{3}$ \\ ${ }^{1}$ School of Medicine, Aja University of Medical Sciences, Tehran, Iran \\ ${ }^{2}$ Lifestyle and Health Management Research Group, Academic Center for Education, Culture and Research (ACECR), Tehran, Iran \\ ${ }^{3}$ Physical therapy Department, School of Rehabilitation, Tehran University of Medical sciences and Health services, Tehran, Iran \\ * Corresponding Author: Manijeh Soleimanifar \\ E-mail: Manijeh.soleimanifar@yahoo.com \\ Tel: +989133037456
}

\begin{abstract}
[Purpose] Although lifting tasks has been recognized as a primary risk factor in low back pain, the concept of lifting asymmetry is relatively new subject. To address trunk function, biomechanical studies generally measure trunk muscle activity using surface electromyography (EMG). But so far, magnitude and similarity index (SI) obtained from EMG have not been studied as indicators of the motor control during lifting task. So, the purpose of this study is to compare the trunk muscles magnitude and SI during symmetric and asymmetric lifting. [Subjects and Methods] A total of 20 healthy male with no history of lumbar spine disorders participated. Surface electromyography data were recorded from the 7 trunk muscles while the participants performed symmetric and asymmetric lifting and lowering different loads. [Results] According to Multivariate ANOVAs the phase of motion (lifting, lowering) and condition (symmetry, asymmetry) have a significant effect on SI and magnitude ( $p \leq 0.05$ ). Load changes have no effect on SI $(p=0.969)$ but have a significant effect on magnitude $(p \leq 0.05)$. The magnitude and $\mathrm{SI}$ value is higher in asymmetrical lifting and lowering compare to symmetrical condition. [Conclusion] The findings reveal the SI value is higher in asymmetric conditions. This means that the amount of muscles cocontracture increased during asymmetrical conditions. Increased muscles co-contracture reinforces the hypothesis of exerting more compression on the spine in asymmetrical movement.

Keywords: Asymmetrical lifting, Motor control, Electromyography
\end{abstract}

\section{INTRODUCTION}

Manual material handling tasks, such as lifting and lowering are considered to be major risk factors in the development of low back pain. ${ }^{1}$ Although lifting has been recognized as a primary risk factor in low back pain, the concept of lifting asymmetry is relatively new subject. $^{2}$ Epidemiological research has shown that when a lifting task is performed asymmetrically there is an increase in the risk of low back pain and intervertebral disc herniation. ${ }^{3}$ It is often argued that this increased risk is due to the increased muscle coactivity, higher compressive and shear forces experienced during asymmetrical lifting activities when compared with symmetrical lifting activities. ${ }^{4}$ But van Dieen and Kingma revealed in a study that the production of asymmetric torque compared to symmetric torque seemed to create less force on the lumbosacral joint and challenged the hypotheses. ${ }^{5}$ Also, in an occupational setting, research has indicated that workers tend to adopt an asymmetric posture when lifting heavier loads, due to the improved mechanical advantage that they receive in these positions. ${ }^{6}$ It seems due to the contradictions raised about how to lift the load (symmetry and asymmetry) and the unclear effect of various weights on trunk muscle activity during lifting and lowering, it is worthwhile to study these cases. To address trunk function, biomechanical studies generally measure trunk muscle activity using surface electromyography (EMG). ${ }^{7}$ In addition to the common methods of assessing muscle activity (onset/ offset, RMS) individually in a specific task, studying all muscles responsible for the entire prototype of a task would be valuable. ${ }^{8}$ In fact, instead of studying each muscle activity individually, the magnitude and the similarity of activities of all muscles involved in a task is studied as a whole in a work pattern. ${ }^{9}$ According to research, Similarity index $(\mathrm{SI})$ is an objective analysis method to evaluate central nervous system (CNS) motor control of voluntary movement. It is a quantitative analysis of EMG data derived from voluntary motor control and provides the quantitative measure of CNS output to muscles. ${ }^{9}$ The magnitude and similarity index were studied in some special cases of the shoulder joint as well as cervical spine and in patients with partial spinal cord injury. ${ }^{8-10}$ But so far this method has not been used to evaluate the trunk muscles activity during manual material handling task. Therefore, the objectives of this study were to investigate the differences in trunk muscles control in lifting and lowering tasks symmetrically and asymmetrically by SI and magnitude.

\section{SUBJECTS AND METHODS}

In this study, 20 healthy male (mean \pm SD $21.25 \pm 1.48$ years, $1.80 \pm 0.06 \mathrm{~m}, 73.05 \pm 8.91 \mathrm{~kg}$ ) with no history of low back pain were examined. Exclusion criteria were back pain and/or neuropathy (sciatica), medication, undergoing psychological treatment, and the existence of any obvious disease. Subjects were recruited randomly from AJA University of Medical Sciences students and workers. The ethical approval code was achieved before conducting the study (IR.AJAUMS.REC.1399.113) and all cases signed an informed consent form.

The EMG activities of the seven trunk muscles that control flexion, extension, and twisting including the right and left erector spine muscles (RES and LES), right and left multifidus muscles (RMF and LMF), right rectus abdominus (RRA), left external oblique (LEO), and right internal oblique (RIO) were recorded during lifting and lowering different loads. The EMG signals were recorded with seven 
pre amplified bipolar active electrodes (Type NO. SX230, Biometrics Ltd, Cwmfelinfach, Gwent, UK) with a fixed center to center inter electrode distance of $20 \mathrm{~mm}$, with a 10 $\mathrm{mm}$ recording diameter, built in differential amplifier with a gain of 1000, input impedance of 1015 Ohms, a common mode rejection ratio of $110 \mathrm{~dB}$ at $60 \mathrm{~Hz}$, bandwidth of 20 $480 \mathrm{~Hz}$, and sampling frequency of $1 \mathrm{KHz}$. Channel sensitivity was 3 microvolts. Reference electrode was placed on the right wrist. Signals were digitally recorded by the data loggers on 256 MB flash memory. The electrodes were placed based on the SENIAM's (Surface Electromyography for the Non-Invasive Assessment of Muscles) recommendations. ${ }^{9}$ The sites of the electrodes place on the skin were cleaned and the electrodes were attached to the skin. The surface electrodes for RES and LES were placed parallel to the $L 3$ level of the spine, $3 \mathrm{~cm}$ lateral from the spine. The electrodes for RMF and LMF were placed parallel to the L5 level of the spine, $2 \mathrm{~cm}$ lateral from the spine. The electrodes for RRA were placed parallel to the muscle fibers of the rectus abdominus such that they were located $2 \mathrm{~cm}$ lateral at the right side of the umbilicus over the muscle belly. The electrodes for LEO were placed $15 \mathrm{~cm}$ lateral at the left side of the umbilicus with a slightly oblique angle such that they ran parallel to the muscle fibers. The electrodes for RIO were placed about halfway between anterior superior iliac spine and symphysis pubis above inguinal ligament. The EMG outcome measures in this study were included the magnitude and the similarity indices of the trunk muscles during lifting and lowering loads symmetrically and asymmetrically. To find these variables, at first, the RMS of each muscle was calculated and called as muscle response ( $R 1, R 2, \ldots, R n)$. Normalize of each muscle response was detected by the vector of ratio between muscle response to rest muscle activity (base line correction, Ri $1,2, \ldots \mathrm{n}$ ) as equation (1).

The response vector $(R V i)$ is summation all $R$ normal $(R 1$ normal, $\mathrm{R} 2$ normal, ..., Rn normal) and prototype response vector is mean of all $R$ normal ( $R 1$ normal, $R 2$ normal, ..., Rn normal). The multiple of RVi and PRVi is named as magnitude $\left(\sum i\right.$ (PVi PRVi).

The Ratio between magnitude of task and magnitude of normal condition is similarity index.

The normal condition was measured by mean deference of each $\mathrm{R}$ normal and then RV and PRV estimated for detection SI, equation (2).

Rnorm $=\frac{[\mathrm{R} 1 \mathrm{R} 2 \mathrm{R} 3 \mathrm{R} 4 \mathrm{R} 5 \mathrm{R} 6 \mathrm{R} 7]}{\sqrt{\sum \mathrm{Ri}^{2}}}$
$\mathrm{SI}=\frac{\sum \mathrm{i}(\mathrm{RVi} \text { PRVi) }}{|\mathrm{RV}||\mathrm{PRV}|}$
The $\mathrm{SI}$ is computed as the inner product between the two vectors where IRVI and IPRVI are the magnitudes of vectors RV and PRV, and RVi represents each element of vector RV.

After installing the electrodes and ensuring proper recording, the subject is placed in front of the target table with $80 \mathrm{~cm}$ height. The distance of the subject from the table was $65 \mathrm{~cm}$ and the distance from the center of the weight box to the line between the subject's medial malleolus was $35 \mathrm{~cm}$. Weights $(5.5,7.5,9.5$, and $12.5 \mathrm{~kg})$ which were calculated based on the revised NIOSH lifting equations ${ }^{11}$ were placed randomly in a plastic box so that participant would not Prejudice about the amount of load. In symmetric lifting and lowering, the subject lifted each weight in the box in position of flexed trunk and hips and extended knees (stoop lifting) and put it on the table. The subject stays in this position for one second and then in reverse action puts the box on the ground in lowering phase. The stoop lifting was purposely chosen as the testing position to not involve muscles of lower and upper extremities. In addition, the selected position significantly reduces the degree of freedom associated with peripheral joints and decreases the kinematic variability. IN the asymmetric lifting and lowering, the weight box is placed on the ground at an angle of 75 degrees to the line perpendicular to the line between the two medial malleolus. With a rotation of 75 degrees relative to the sagittal plane, the subject lifted the weight from the ground and places it on the table, and after a second, returned it to its original position on the ground in lowering phase. Lifting and lowering tasks were performed 3 times for each weight in each condition. Each trial began as soon as the participant received visual feedback. In order to obtain more information for motion sequence, the physiotherapist divided the experiments into two phases of lifting and lowering with the event marker, which was synchronized with the EMG. To minimize the effects of fatigue, the participants rested for 5 minutes between each trial. The entire procedure was carried out by 7 years experienced well-trained physical therapist with expertise in EMG and bio-mechanic.

For statistical comparisons, EMG parameters (magnitude and $\mathrm{SI}$ ) were averaged over the 3 trials. In order to analyze the statistical findings, first, descriptive statistics were presented. Multivariate ANOVA was used to detect the effect of task phase (lifting, lowering) condition (symmetry, asymmetry) and loads $(5.5,7.5,9.5,12.5 \mathrm{~kg})$ on SI and magnitude. Paired-samples t-test was used to examine the effect of symmetry and asymmetry on the SI and the Magnitude separately while lifting and lowering weights. Statistical analyses were performed using SPSS software (ver. 18.0 for Windows; SPSS Inc., Chicago, IL, USA), and statistical significance was set at $p \leq 0.05$. 


\section{RESULTS}

Table 1 shows mean \pm SD of SI and magnitude in different loads, motion phase (lifting, lowering) and condition (symmetry, asymmetry).

\begin{tabular}{|l|c|c|c|c|c|}
\hline \multirow{3}{*}{ Weight in box } & \multirow{2}{*}{$\begin{array}{c}\text { Dependent } \\
\text { variable }\end{array}$} & \multicolumn{2}{|c|}{ lifting } & \multicolumn{2}{c|}{ lowering } \\
\cline { 2 - 6 } & & symmetry & asymmetry & symmetry & asymmetry \\
\hline $\mathbf{5 . 5} \mathbf{~ k g}$ & $\mathrm{SI}$ & $0.37 \pm 0.03$ & $0.40 \pm 0.04$ & $0.43 \pm 0.06$ & $0.47 \pm 0.09$ \\
\cline { 2 - 6 } & $\mathrm{Mag}$ & $114.21 \pm 37.45$ & $123.20 \pm 42.64$ & $86.87 \pm 28.59$ & $96.04 \pm 31.93$ \\
\hline $\mathbf{7 . 5} \mathbf{~ k g}$ & $\mathrm{SI}$ & $0.37 \pm 0.04$ & $0.41 \pm 0.05$ & $0.41 \pm 0.06$ & $0.46 \pm 0.07$ \\
\cline { 2 - 6 } & $\mathrm{Mag}$ & $123.15 \pm 40.19$ & $128.94 \pm 43.10$ & $90.38 \pm 30.65$ & $102.17 \pm 40.52$ \\
\hline $\mathbf{9 . 5} \mathbf{~ k g}$ & $\mathrm{SI}$ & $0.38 \pm 0.04$ & $0.41 \pm 0.05$ & $0.44 \pm 0.07$ & $0.47 \pm 0.07$ \\
\cline { 2 - 6 } & $\mathrm{Mag}$ & $126.47 \pm 43.34$ & $142.44 \pm 57.05$ & $96.71 \pm 35.52$ & $107.97 \pm 40.03$ \\
\hline $\mathbf{1 2 . 5} \mathbf{~ k g}$ & $\mathrm{SI}$ & $0.38 \pm 0.04$ & $0.42 \pm 0.04$ & $0.42 \pm 0.06$ & $0.47 \pm 0.08$ \\
\cline { 2 - 6 } & $\mathrm{Mag}$ & $138.81 \pm 47.18$ & $152.14 \pm 53.77$ & $107.22 \pm 41.65$ & $116.16 \pm 42.91$ \\
\hline
\end{tabular}

Note: $\mathrm{SI}=$ similarity Index, Mag= Magnitude

Table 2 shows the results of the Multivariate ANOVAs to evaluate the effect of motion phase (lifting, lowering),

condition (symmetry, asymmetry) and loads (5.5, 7.5, 9.5, $12.5 \mathrm{~kg}$ ) on SI and magnitude.

Table 2. Multivariate ANOVAs to detect differences between task phase (lifting, lowering), condition (symmetry, asymmetry) and loads (5.5, $7.5,9.5,12.5 \mathrm{~kg}$ ) and their interaction.

\begin{tabular}{|c|c|c|c|c|c|}
\hline Source of variation & $\begin{array}{l}\text { Dependent } \\
\text { variable }\end{array}$ & $\begin{array}{l}\text { Sum of } \\
\text { square }\end{array}$ & Mean square & $\mathbf{F}$ & P-value \\
\hline \multirow[t]{2}{*}{ Task phase } & $\mathrm{SI}$ & 0.391 & 0.391 & 99.663 & $0.000^{*}$ \\
\hline & Mag & 110734.055 & 110734.055 & 55.379 & $0.000^{*}$ \\
\hline \multirow[t]{2}{*}{ condition } & SI & 0.152 & 0.152 & 38.715 & $0.000^{*}$ \\
\hline & Mag & 14443.990 & 14443.990 & 7.223 & $0.007^{*}$ \\
\hline \multirow[t]{2}{*}{ load } & $\mathrm{SI}$ & 0.001 & 0.000 & 0.084 & 0.969 \\
\hline & Mag & 39422.901 & 13140.967 & 6.572 & $0.000^{*}$ \\
\hline \multirow[t]{2}{*}{ Task phase ${ }^{\star}$ condition } & $\mathrm{SI}$ & 0.007 & 0.007 & 1.858 & 0.173 \\
\hline & Mag & 57.576 & 57.576 & 0.029 & 0.865 \\
\hline \multirow[t]{2}{*}{ Task phase ${ }^{\star}$ load } & SI & 0.002 & 0.001 & 0.205 & 0.893 \\
\hline & Mag & 1047.653 & 349.218 & 0.175 & 0.914 \\
\hline \multirow[t]{2}{*}{ Condition*load } & SI & 0.005 & 0.002 & 0.418 & 0.740 \\
\hline & Mag & 426.572 & 142.191 & 0.071 & 0.975 \\
\hline \multirow{2}{*}{$\begin{array}{c}\text { Task } \\
\text { phase*condition`load }\end{array}$} & $\mathrm{SI}$ & 0.002 & 0.001 & 0.187 & 0.906 \\
\hline & Mag & 78.300 & 26.100 & 0.013 & 0.998 \\
\hline
\end{tabular}

Note: * significant difference between different task phase, different condition and different loads, $p \leq 0.05$

$\mathrm{SI}=$ similarity Index, Mag= Magnitude

According to table 2 the phase of motion (lifting, lowering) and condition (symmetry, asymmetry) have a significant effect on $\mathrm{SI}$ and magnitude $(\mathrm{p} \leq 0.05)$. Load changes have no effect on SI $(p=0.969)$ but have a significant effect on magnitude $(p \leq 0.05)$. The interaction of source of variation on each other was not significant.

The SI and magnitude values were calculated for 5.5, 7.5, 9.5 , and $12.5 \mathrm{~kg}$ loads during lifting and lowering in 


\begin{tabular}{|c|c|c|c|c|c|c|c|}
\hline \multirow{2}{*}{$\begin{array}{l}\text { weight } \\
\text { in box }\end{array}$} & \multirow{2}{*}{$\begin{array}{l}\text { Dependen } \\
\text { t variable }\end{array}$} & \multicolumn{3}{|c|}{ lifting } & \multicolumn{3}{|c|}{ lowering } \\
\hline & & $\begin{array}{c}\text { Symmetry/asymmetry } \\
\text { mean difference }\end{array}$ & SD & $\mathrm{P}$ value & $\begin{array}{c}\text { Symmetry/asymmetry } \\
\text { mean difference }\end{array}$ & SD & $P$ value \\
\hline \multirow[t]{2}{*}{$5.5 \mathrm{~kg}$} & SI & -0.02 & 0.03 & $0.02^{*}$ & -0.03 & 0.07 & $0.04^{*}$ \\
\hline & Mag & -8.99 & 27.32 & 0.15 & -9.16 & 12.88 & $0.00^{*}$ \\
\hline \multirow[t]{2}{*}{$7.5 \mathrm{~kg}$} & $\mathrm{SI}$ & -0.03 & 0.04 & $0.00^{*}$ & -0.04 & 0.06 & $0.00^{*}$ \\
\hline & Mag & -5.75 & 29.30 & 0.38 & -11.78 & 14.73 & $0.00^{*}$ \\
\hline \multirow[t]{2}{*}{$9.5 \mathrm{~kg}$} & SI & -0.03 & 0.05 & $0.00^{*}$ & -0.03 & 0.05 & $0.01^{*}$ \\
\hline & Mag & -15.97 & 32.61 & 0.06 & -11.26 & 16.84 & $0.00^{*}$ \\
\hline \multirow[t]{2}{*}{$12.5 \mathrm{~kg}$} & $\mathrm{SI}$ & -0.03 & 0.03 & $0.00^{*}$ & -0.05 & 0.05 & $0.00^{*}$ \\
\hline & Mag & -13.32 & 32.22 & 0.08 & -8.93 & 15.09 & $0.01^{*}$ \\
\hline
\end{tabular}

Note: *significant difference between different condition in different phase, $p \leq 0.05$

$\mathrm{SI}=$ similarity Index, Mag= Magnitude

Tables 3 shows that when lifting loads asymmetrically, the amount of $\mathrm{SI}$ increases significantly compared to the symmetrical condition $(p \leq 0.05)$. But the magnitude was not significantly different between symmetric and asymmetric lifting. The mean of the magnitude increased in both condition with increasing load.

Tables 3 shows that when lowering loads asymmetrically, the amount of $\mathrm{SI}$ and magnitude increases significantly compared to the symmetrical condition $(p \leq 0.05)$.

\section{DISCUSSION}

The present study is one of the first studies to compare the trunk muscles control during symmetric and asymmetric lifting by $\mathrm{SI}$ and magnitude. The SI represents the distribution of the EMG pattern. In this study, if the ratio of agonist and antagonist muscles involvement in a specific task is similar to each other, $\mathrm{SI}$ is closer to one.

Lifting Vs. lowering: The results showed that the magnitude in the lifting phase is more than lowering phase. And the SI value in lowering phase is higher than lifting phase. The muscles activities and the resultant forces on the back during lifting and lowering weights were studied and compared in some earlier investigations. Researchers have found that the activity of the back extensor muscles was lower for lowering tasks than for lifting tasks. ${ }^{12-13}$ Most studies have attributed these differences to force-velocity relationship of muscle. During concentric contractions of the erector spine during lifting, the capacity to generate force is decreased relative to an isometric contraction. Conversely, there is an enhancement in force generating capacity during eccentric contractions associated with lowering ${ }^{13}$ However, given the effects of contraction velocity on force generating capacity, the neural drive required during lifting would have to be higher than that during lowering the moments. As a result, more extensor muscles firing rate during lifting phase leads to an increase in EMG magnitude. It has been commonly found that the lowering strength is greater than lifting strength. ${ }^{14-15}$ Davis et al. have declared lowering strength is to be $56 \%$ greater than lifting strength ${ }^{15}$. Considering the more strength and less back activities required for lowering and also more coordination of agonist and antagonist muscles in the trunk, the lowering phase has a better neuromuscular control than the lifting phase. Therefore, higher SI value in lowering phase it seems logical.

Symmetry vs. asymmetry: The results revealed that the magnitude trend is increasing in the asymmetric condition compared to symmetric, but this increase was significant only in lowering phase. Also, the SI value is higher in asymmetrical lifting and lowering compare to symmetrical condition. Spinal compression forces are mainly determined by muscle forces, required to overcome moments caused by gravity. In addition to the magnitude of the torque, the direction of the torque resulting from the muscle contractions affects the amount of compressive force applied to the spine. There are hypotheses that in asymmetric lifting more compressive forces are exerted on the lumbar spine. In the asymmetric state, the torques resulting from the contraction of the trunk muscles are applied orthopedically in several axes (more than one axis). To prevent fatigue, the participation of more muscle groups in asymmetric lifting leads to more load on the spine ${ }^{16}$. Moreover, people find asymmetric lifting to be a more challenging condition ${ }^{17}$ and consequently increase torso stiffness due to muscle co-contraction. The findings of the present study seem to confirm these hypotheses. Muscles co-contractions in asymmetric activity lead to increased SI and magnitude in this condition. Biomechanical studies have shown that maximum trunk strength, maximum acceptable weight, and pattern of trunk muscles activity are significantly affected in asymmetric postures ${ }^{2}$. Despite the reduction in trunk torque production capacity in asymmetric activities, muscle recruitment in combined activities will be greater than symmetric activity. It seems that in asymmetric conditions, due to the shortening of the muscular lever arm, the activity of the muscles increase to produce the required force.

Loads $(5.5,7.5,9.5,12.5 \mathrm{~kg})$ : The results showed that increasing the load case increases the trunk muscles 
magnitude. This finding is obvious, because the neuromuscular system increases its activity under the influence of increased load by two mechanisms which include increases motor unit firing rate and new motor unit recruitment ${ }^{18}$. The results also showed that load changes did not affect the SI in the mentioned muscles. The nature of the $\mathrm{SI}$ is such that it has not related the quantity amount of muscle activity. In fact, this index indicates a correlation in the direction of changes and the degree of dispersion of the set of muscles involved in a motor function. Therefore, according to the obtained results, the main function of the muscles and the ratio of their changes did not differ from the load changes. In other words, with increasing load, the contribution of muscle activity increased proportionately and the SI did not change.

\section{CONCLUSION}

The magnitude during lifting is greater than lowering, which is probably due to the type of muscle contraction. This increase can predict the possibility of damage to the spine when carrying heavy objects during the lifting phase. The findings reveal the SI value is higher in asymmetric conditions. This means that the amount of muscles cocontracture increased during asymmetrical conditions. Increased muscles co-contracture reinforces the hypothesis of exerting more compression on the spine in asymmetrical movement.

\section{Acknowledgements}

We appreciate the AJA University of Medical Sciences, for providing the generous grant to carry out this research.

\section{REFERENCES}

1. de Looze MP, Kingma I, Thunnissen W, van Wijk MJ, Toussaint HM. The evaluation of a practical biomechanical model estimating lumbar moments in occupational activities, Ergonomics. 1994; 37: 1495-1502.

2. Cole M, Grimshaw P. Low back pain and lifting: A review of epidemiology and aetiology. Work (Reading, Mass.). 2003; 21: 173-84.

3. Kingma I, van Dieën JH, de Looze MP, Toussaint HM, Dolan $\mathrm{P}$, Baten CTM. Asymmetric low back loading in asymmetric lifting movements is not prevented by pelvic twist, Journal of Biomechanics. 1998; 31: 527-534.

4. Marras WS, Davis KG. Spine loading during asymmetric lifting using one versus two hands, Ergonomics. 1998; 41: 817-834.

5. van Dieën JH, Kingma I. Total trunk muscle force and spinal compression are lower in asymmetric moments as compared to pure extension moments, Journal of Biomechanics. 1999; 32: 681687

6. Gagnon M, Plamondon A, Gravel D. Pivoting with the load: an alternative for protecting the back in asymmetrical lifting, Spine. 1993; 18: 1515-1524.

7. De Luca CJ, Adam A, Wotiz R, Gilmore LD, Nawab SH. Decomposition of surface EMG signals. J Neurophysiol. 2006; 96:1646-57

8. Lin JJ, Lim HK, Soto-quijano DA, Hanten WP, Olson SL, Roddey TS, et al. Altered patterns of muscle activation during performance of four functional tasks in patients with shoulder disorders: interpretation from voluntary response index. J Electromyogr Kinesiol. 2006; 16:458-68.

9. Ghofrani M, Olyaei G, Talebian S, Bagheri H, Kazemi P. Reliability of SEMG measurements for trunk muscles during lifting variable loads in healthy subjects. J Bodyw Mov Ther. 2017l; 21:711-718. doi: 10.1016/j.jbmt.2016.12.003. Epub 2016 Dec 10. PMID: 28750989.

10. Cheng $\mathrm{CH}$, Lin $\mathrm{KH}$, Lin JJ, Wang JL. Effect of movement speed on cervical muscle activation pattern in healthy adults: voluntary response index analysis. J Biomechanic. 2007; 40: S413

11. Waters TR, Putz-Anderson V, Garg A, Fine LJ. Revised $\mathrm{NIOSH}$ equation for the design and evaluation of manual lifting tasks. Ergonomics. 1993; 39: 749-776

12. De Looze MP, Toussaint HM, Van Dieen JH, Kemper HCG. Joint moments and muscle activity in the lower extremities and lower back in lifting and lowering tasks. Journal of Biomechanics. 1993; 26: 1067-1076

13. McKean CM, Potvin JR. Effects of a simulated industrial bin on lifting and lowering posture and trunk extensor muscle activity. International Journal of Industrial Ergonomics. 2001; 28: $1-15$

14. Marras WS, Mirka GA. A comprehensive evaluation of trunk response to asymmetric trunk motion. Spine. 1992; 17: 253379

15. Davis KG, Marras WS, Waters TR. Evaluation of spinal loading during lowering and lifting. Clin Biomech. 1998; 13: 141-152

16. DieeKn van JH. Preliminary validation of a model to estimate tissue loads in the lumbosacral spine. In: Kumar, S. (Ed.) Advances in Occupational Ergonomics and Safety. IOS, Amsterdam, 1998. 275-278.

17. Mital A, Fard F. Psychophysical and physiological responses to lifting symmetrical and asymmmetrical loads symmetrically and asymmetrically. Ergonomics. 1986; 29: 1263-1272,

18. Lindner T, Schulze C, Woitge S, Finze S, Mittelmeier W, Bader R. The Effect of the Weight of Equipment on Muscle Activity of the Lower Extremity in Soldiers. The Scientific World Journal. 2012. vol. 2012, Article ID 976513, 8 pages, https://doi.org/10.1100/2012/976513 